\title{
Tuberous sclerosis complex presenting as periungual fibromas and seizures in a 52-year-old woman
}

\author{
Daniel Ortega-Quijano MD, Bibiana Pérez-García MD, Sergio Vañó-Galván PhD MD
}

Cite as: CMAJ 2020 June 29;192:E714. doi: 10.1503/cmaj.190449

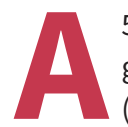

52-year-old woman presented to our dermatology clinic with progressively enlarging skin-coloured excrescences on several fingers (Figure 1A). She had a long-standing history of well-controlled seizures (focal onset aware) that had begun in adolescence. She was otherwise asymptomatic. There was no family history of genodermatosis. Given the combination of seizures and skin findings consistent with periungual fibromas, we considered the possibility of tuberous sclerosis complex (TSC).

The initial workup included brain magnetic resonance imaging, which showed a hyperintense focus on $T_{2}$ and fluid-attenuated inversion recovery (FLAIR) in cortical and subcortical white matter, compatible with dysplastic foci from TSC (Figure 1B). Screening for other manifestations of the condition (ophthalmic, pulmonary, cardiologic and nephrologic) was unremarkable. Genetic analysis showed a sporadic mutation of the TSC1 gene. The patient received carbon-dioxide laser treatment and surgery for the skin lesions, and is monitored regularly for complications related to TSC.

Tuberous sclerosis complex is an autosomal dominant genetic disorder with a birth incidence of 1:5800. ${ }^{1}$ Hamartomas (benign tumours) can develop in any organ system. The classic triad of seizures, intellectual disability and skin findings usually presents early in life in those who are affected, but only $30 \%$ of patients have all 3 characteristics, $6 \%$ have none and $12 \%$ receive their diagnosis in adulthood. ${ }^{2}$

Periungual fibromas affect up to $50 \%$ of people with TSC. They present as multiple slowly growing, elongated or oval, flesh-coloured lesions with distal hyperkeratosis. ${ }^{3}$ They can lead to distortion of the nail plate; both fingers and toes may be affected. Various surgical, laser and chemical ablation techniques have been described with variable long-term results.

Morbidity and mortality in TSC mainly result from pulmonary (lymphangiomyomatosis) and renal disease (angiomyolipomas and renal cysts). Early treatment directed to renal and lung manifestations with a mammalian target of rapamycin inhibitor or surgery may improve prognosis. ${ }^{1}$

\section{References}

1. Krueger DA, Northrup H; International Tuberous Sclerosis Complex Consensus Group. Tuberous sclerosis complex surveillance and management: recommendations of the 2012 International Tuberous Sclerosis Complex Consensus Conference. Pediatr Neurol 2013;49:255-65.

2. Gomez MR. Tuberous sclerosis. 2nd ed. New York: Raven Pr; 1988.

3. Thomas L, Zook EG, Haneke E, et al. Chapter 13: Tumors of the nail apparatus and adjacent tissues. In: Baran and Dawber's Diseases of the Nails and their Management, Fourth Edition. John Wiley \& Sons, Ltd.; 2008:637-743. Available: https://doi.org/10.1002/9781118286715. ch13 (accessed 2019 Nov. 20).

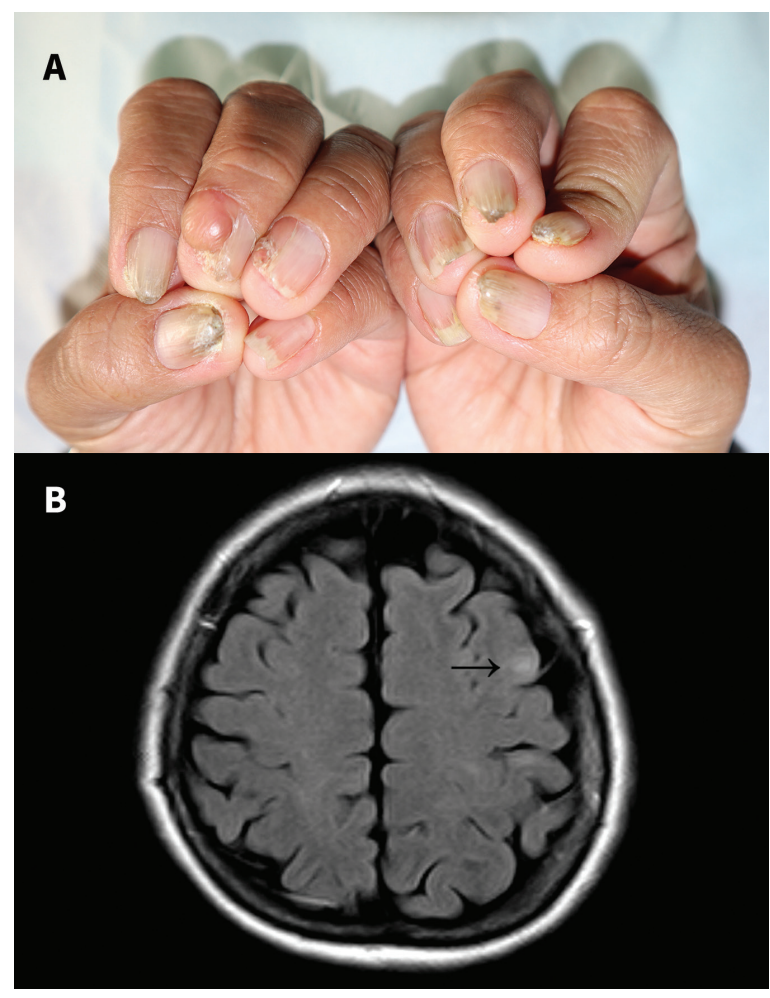

Figure 1: (A) Skin-coloured tumours and secondary nail dystrophy, consistent with periungual fibromas, on several fingers in a 52-year-old woman with seizures. (B) Brain magnetic resonance image from a fluid-attenuated inversion recovery sequence showing a hyperintense subcortical white matter area (arrow) compatible with a dysplastic focus in the context of tuberous sclerosis complex.

Competing interests: None declared.

This article has been peer reviewed.

The authors have obtained patient consent.

Affiliation: Servicio de Dermatología, Hospital Universitario Ramón y Cajal, Instituto Ramón y Cajal de Investigación Sanitaria (IRYCIS), Madrid, Spain

Correspondence to: Daniel Ortega-Quijano, Daniel-oq@ hotmail.com 